\title{
Ácaros ectoparasitos de morcegos no estado de Mato Grosso do Sul, Brasil
}

\author{
Camila de Lima Silva', Michel P. Valim² \& Gustavo Graciolli’
}

\author{
1. Centro de Ciências Biológicas e da Saúde, Universidade Federal de Mato Grosso do Sul, Caixa Postal 549, 79070-900 Campo Grande, MS, Brasil. \\ (camila_bio@hotmail.com) \\ 2. Museu de Zoologia da Universidade de São Paulo, Serviço de Invertebrados, Av. Nazaré, 481, Ipiranga, $04263-000$ São Paulo, SP, Brasil.
}

Recebido 7 dezembro 2016

Aceito 6 fevereiro 2017

DOI: $10.1590 / 1678-4766 e 2017111$

\begin{abstract}
Bat mites and bat chiggers of the state of Mato Grosso do Sul, Brazil. Up to now, 49 species of bat mites and chiggers are recorded in Brazil, divided into ten families and three suborders. To verify the occurrence of the bat mites and chiggers species in the state of Mato Grosso do Sul, specimens deposited at the "Coleção Zoológica de Referência da Universidade Federal de Mato Grosso do Sul” were examined and queried information available in literature. It was found 25 species of these mites, belonging to six families from bats in Mato Grosso do Sul. All species are a new geographic record for the state. Additionally, a checklist of bat mites and chiggers, with their respective hosts and states that occur in Brazil is presented.
\end{abstract}

KEYWORDS: Biodiversity, Biota-MS program, Pantanal, parasitism.

RESUMO. Atualmente, 49 espécies de ácaros associadas a morcegos distribuídas em dez famílias e três subordens são registradas no Brasil. Para verificar ocorrência das espécies de ácaros ectoparasitos de morcegos no estado de Mato Grosso do Sul, foram revisados espécimes depositados na Coleção Zoológica de Referência da Universidade Federal de Mato Grosso do Sul e consultadas informações disponíveis na literatura. Foram registradas 25 espécies de ácaros de seis famílias e três subordens no Mato Grosso do Sul. Todas as espécies constituem novo registro geográfico para o estado. Adicionalmente é apresentada uma listagem das espécies de ácaros ectoparasitos de morcegos, com seus respectivos hospedeiros e estados em que ocorrem no Brasil.

PALAVRAS-CHAVE: Biodiversidade, Programa Biota-MS, Pantanal, parasitismo.

Os morcegos são excelentes modelos para o estudo da relação hospedeiro-parasito, principalmente seus artrópodes parasitos. Não apenas pela diversidade taxonômica, mas também por possuírem diferentes hábitos, os quirópteros albergam uma rica fauna de ectoparasitos. A comunidade de artrópodes ectoparasitos que ocorre em morcegos são (1) insetos Siphonaptera (pulgas), Diptera (moscas), Hemiptera (percevejos) e Dermaptera (tesourinhas) e (2) aracnídeos pertencentes ao grupo dos ácaros Sarcoptiformes, Trombidiformes, Mesostigmata e Ixodida, todas ordens da subclasse Acari associados com hospedeiros vertebrados encontrados nos Chiroptera. Algumas dessas ordens possuem famílias encontradas exclusivamente em morcegos (e.g. Labidocarpidae, Spinturnicidae e Spelaeorhynchidae). Aproximadamente 1.400 espécies de ácaros (KRANTZ \& WALTER, 2009), enquanto que apenas cerca de 700 de insetos foram descritas sobre morcegos (WEBB JR. \& LoOMIS, 1977). Esse valor duas vezes maior reflete a diversidade de formas, hábitos e micro-hábitats proporcionados pelo corpo desses hospedeiros, resultando uma grande diversidade de ácaros associados à ordem Chiroptera.
Os ácaros ectoparasitos podem passar a vida inteira no corpo do seu hospedeiro (parasito permanente), alimentandose continuamente, ou permanecem nos seus abrigos, se nutrindo em períodos de inatividade do hospedeiro (parasito temporário) (MARSHALL, 1981). Os ectoparasitos permanentes são geralmente menores que os temporários e apresentam preferência por lugares específicos do corpo hospedeiro. $\mathrm{O}$ conhecimento desses hábitos facilita o estudo prévio para que as coletas sejam otimizadas, uma vez que se sabe o que e onde capturar.

O estudo sobre a composição das comunidades de ácaros ectoparasitos e os padrões de infestação é ponto fundamental para a compreensão da ecologia do ectoparasitismo em morcegos. Apenas recentemente alguns estudos foram feitos no Brasil inventariando a fauna regional de ácaros associados a morcegos (GETTINGER \& GRIBEL, 1989; AzEvedo et al., 2002; DANTAs-Torres et al., 2009; Silva et al., 2009; AlmeIdA, et al., 2011).

Atualmente são registradas no Brasil 49 espécies de ácaros associadas a morcegos, distribuídas em dez famílias e três subordens. Essas famílias apresentam distintas 
adaptações morfológicas, hábitos alimentarem e preferências por hospedeiros.

\section{MATERIAL E MÉTODOS}

A listagem foi feita a partir de espécimes depositados na Coleção Zoológica de Referência da Universidade Federal de Mato Grosso do Sul (ZUFMS), Campo Grande, Mato Grosso do Sul, Brasil, e dados disponíveis em artigos científicos, dissertações e teses. Os artigos foram pesquisados nas seguintes bases de dados: Scielo (www.scielo.org), Scopus (www.scopus.com) e Web of Knowledge (http:// apps.webofknowledge.com) até data de 15 de janeiro de 2013.

$\mathrm{Na}$ listagem apresentada, as subordens estão em ordem filogenética (grupo basal para o mais apical) e, em cada subordem as famílias, assim como os gêneros e as espécies, estão em ordem alfabética. Para as espécies dos hospedeiros seguiu-se GARDNER (2008), sendo que os nomes subespecíficos não foram utilizados. No item material examinado as informações são apresentadas no seguinte ordem: sigla da localidade, números de indivíduos da espécie de ácaro e data de coleta.

\section{RESULTADOS E DISCUSSÃO}

$\mathrm{Na}$ literatura consultada não foram encontradas informações sobre a ocorrência de ácaros ectoparasitos de morcegos no estado de Mato Grosso do Sul. Na ZUFMS foram registrados indivíduos de cinco famílias provenientes de três regiões localizadas no bioma Pantanal desse estado: (1) BEP, Corumbá, Passo do Lontra, Base de Estudos do Pantanal da Universidade Federal de Mato Grosso do Sul (19³4'37’S; 5700'42”W); (2) FRN, Aquidauana, Fazenda Rio Negro (19³4'22”S; 56¹4’36”W); FSB, Corumbá, Passo do Lontra, Fazenda São Bento (19²9’25,35”S; 5700'21,71’W).

Mesostigmata

Macronyssidae

Chiroptonyssus haematophagus (Fonseca, 1935)

Material examinado. FSB, Molossus rufus, ô, 24 protoninfas, 26-28.II.2011; Eumops dabennei, 3 protoninfas, 28.I.2011; BEP, M. rufus, 2 + , 62 protoninfas, 25.IV.2011, 27 28.IV.2011; Molossus molossus, ô, 5 protoninfas, 25.IV.2011.

\section{Chiroptonyssus robustipes (Ewing, 1925)}

Material examinado. BEP, Noctilio albiventris, 9,1 protoninfa, 24.VI.2011.

\section{Macronyssoides kochi (Fonseca, 1948)}

Material examinado. FSB, Artibeus planirostris, 12 protoninfas, 29.I.2011, 01.III.2011, 24.IV.2011, 25.V.2011; Platyrrhinus lineatus, 2 protoninfas, 01.III.2011, 27.V.2011; Sturnira lilium, 1 protoninfa, 24.V.2011; BEP, P. lineatus,
5 protoninfas, 29.V.2011, 24.VI.2011; A. planirostris, 2 , 28.VI.2011.

\section{Macronyssoides sp.}

Material examinado. FSB, Artibeus planirostris, 3 protoninfas, 28.I.2011.

\section{Macronyssus meridionalis Radovksi, 1967}

Material examinado. BEP, Myotis nigricans, 1 protoninfa, 27.IV.2011.

\section{Macronyssus sp. 1}

Material examinado. FSB, Chrotopterus auritus (Peters, 1856), 2犬, 6 protoninfas, 26.II.2011.

\section{Macronyssus sp. 2}

Material examinado. FSB, Myotis nigricans, 2우, 18 protoninfas, 26.II.2011; BEP, M. nigricans, $3 \hat{\jmath}$, 10 9 , 1 protoninfa, 24.VI.2011, 28.VI.2011.

\section{Mitonyssus noctilio Yunker \& Radovsky, 1980}

Material examinado. FSB, Noctilio albiventris, 9 , 27.I.2011; BEP, N. albiventris, + , 1 protoninfa, 25.IV.2011.

\section{Parichoronyssus sp.}

Material examinado. FSB, Phyllostomus hastatus, ग, 25.V.2011.

Radfordiella desmodi Radovsky, 1967

Material examinado. FSB, Desmodus rotundus, $5 \hat{\jmath}$, 6 , 25.V.2011.

\section{Radfordiella oudemansi Fonseca, 1948}

Material examinado. BEP, Phyllostomus discolor,

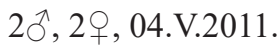

\section{Steatonyssus joaquimi (Fonseca, 1935)}

Material examinado. FSB, Artibeus planirostris, ㅇ, , 2./ II.2011; BEP, Myotis nigricans, 5 +, 1 protoninfa, 28.VI.2011.

$$
\text { Spinturnicidae }
$$

\section{Periglischrus acutisternus Machado-Allison, 1964}

Material examinado. FSB, Phyllostomus hastatus (Pallas, 1767), 2犬, 25.V.2011; BEP, Phyllostomus discolor 


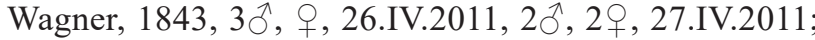
$3 \hat{\jmath}$, 28.IV.2011; 4仓े, 04.V.2011; FRN, P. discolor, $3 \hat{\jmath}, 1$ ninfa, I.2004.

\section{Periglischrus caligus Kolenati, 1857}

Material examinado. FRN, Artibeus planirostris, 우, VI.2003; Glossophaga soricina (Pallas, 1766), 6ð̄, 2 ninfas, III.2003, IX/2004.

\section{Periglischrus herrerai Machado-Allison, 1965}

Material examinado. FSB, Desmodus rotundus (E. Geoffroy, 1810), 7ð̂, o, 26.II.2011, 28.II.2011, 25.V.2011, 28.V.2011; FRN, Artibeus planirostris, 4ð̄, q, VII.2003; D. rotundus, 7ðे, + , 3 ninfas, III.2003; Sturnira lilium (E. Geoffroy, 1810), $3 \hat{\jmath}$, IX.2004.

\section{Periglischrus iheringi Oudemans, 1902}

Material examinado. FSB, Artibeus planirostris, 351ð, 245 9, 60 ninfas, 27.I.2011, 28.I.2011, 29.I2011, 30.I.2011, 26-28.II.2011, 01.III.2011, 30.IV.2011, 01.V.2011, 24-28.V.2011, 23.VI.2011, 25.VI.2011); Chiroderma doriae Thomas, 1891, 4§े, q, 25.VI.2011, 29.VI.2011; Platyrrhinus lineatus, 41ô, 44ㅇ, 1 ninfa, 29-30.I.2011, 28.II.2011, 01.III.2011, 01.V.2011, 27-28.V.2011, 25.VI.2011; Sturnira

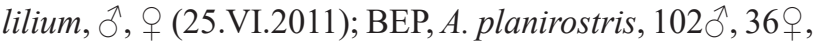
10 ninfas, 31.I.2011, 25.II.2011, 25-26.IV.2011, 28-29. IV.2011, 03.V.2011, 29.V.2011, 28.VI.2011); P. lineatus, $150 \widehat{\jmath}, 105$, 12 ninfas, 31.I.2011, 25.II.2011, 28.IV.2011, 0204.V.2011, 29.V.2011, 24.VI.2011, 26.VI.2011, 28.VI.2011; FRN, Noctilio albiventris, $2 \hat{\jmath},+$, A . planirostris, $131 \hat{\jmath}, 28$, 28 ninfas (2003, 2004); Artibeus lituratus (Olfers, 1818), $15 \hat{\text { A }, ~} 5$ 9, 4 ninfas (III.2003, I.2004, IV.2004); Lophostoma silviculum d'Orbigny, 1836, ô, +, IV.2004; Phyllostomus

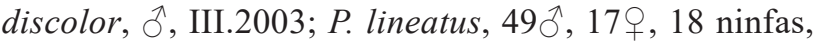
VI.2002, II-III.2003, I.2004, IV.2004, IX.2004); S. lilium, $12 \hat{\jmath}$, ㅇ, I.2003, III.2003.

\section{Periglischrus ojastii Machado-Allison, 1964}

Material examinado. FSB, Sturnira lilium, 25.VI.2011; FRN, S. lilium, 34仓̊, 4ㅇ, III.2003, IX.2004.

\section{Periglischrus tonatii Herrin \& Tipton, 1975}

Material examinado. FSB, Lophostoma silviculum, ^, ㅇ, 30.I.2011; FRN, L. silviculum, 26ð, 25, 6 ninfas, III.2003, VIII.2003, I.2004, IV.2004, IX.2004.

\section{Periglischrus torrealbai Machado-Allison, 1965}

Material examinado. FSB, Phyllostomus hastatus, 50̂, 5 9 , 25.V.2011; BEP, Phyllostomus discolor, 3ðे, 26-27.

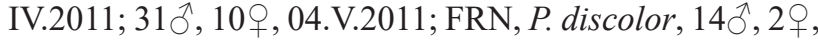
2 ninfas, IX.2004; P. hastatus, $2 \widehat{\jmath}$, VI.2003; Lophostoma silviculum, ㅇ, VIII.2003.

\section{Spinturnix americanus (Banks, 1902)}

Material examinado. FSB, Myotis albescens (E.

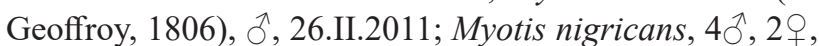
29-30.I.2011, 26.II.2011; Myotis sp., 20, o , 26.V.2011; BEP, Myotis simus Thomas, 1901, 28 đ̂, 17ㅇ, 27.IV.2011;

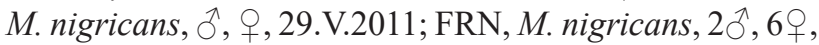
III.2003, I.2004.

\section{Trombidiformes Myobiidae \\ Eudusbabekia sp.}

Material examinado. FSB, Artibeus planirostris, 1 ninfa, 27.V.2011.

\section{Trombiculidae \\ Beamerella sp.}

Material examinado. FSB, Artibeus planirostris, 5 larvas.

\section{Sarcoptiformes \\ Chirodiscidae \\ Parakosa flexipes (Pinichpongse, 1963)}

Material examinado. FSB, Molossus rufus E. Geoffroy, 1805, ふ̋, 16우, 11 ninfas, 28.I.2011, 26.II.2011.

\section{Parakosa rectipes (Pinichpongse, 1963)}

Material examinado. FSB, Cynomops abrasus (Temminck, 1827), vários indivíduos de todos os estádios, 26.II.2011; Molossus rufus, vários indivíduos de todos os estádios, 28.I.2011, 26.II.2011; BEP, Molossus molossus (Pallas, 1766), vários indivíduos de todos os estádios, 25.IV.2011, M. rufus, vários indivíduos de todos os estádios, 26.IV.2011.

Sarcoptidae

\section{Chirnyssoides aff. caparti}

Material examinado. FSB, Artibeus planirostris, 2§, 26-27.II.2011; BEP, A. planirostris, 3ㅇ, 29.V.2011, 28.VI.2011.

O conhecimento sobre a distribuição geográfica de ácaros ectoparasitos de morcegos e seus hospedeiros é escasso no Brasil; somente em 15 estados (dos 26) e no Distrito Federal há registros desses grupos de ácaros (Tab. I).

Apesar de apenas três localidades no estado do Mato Grosso do Sul possuírem ocorrência de ácaros ectoparasitos de morcegos, é o estado com maior número de espécies 
Tab. I. Espécies de ácaros encontradas sobre morcegos no Brasil, seus hospedeiros e distribuição geográfica por estado e Distrito Federal. Fontes: ${ }^{1}$,

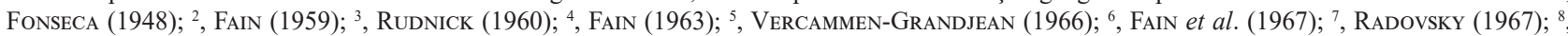
Vercammen-Grandjean (1967); ${ }^{9}$, Brennan (1969); ${ }^{10}$, Fain \& Aitken (1969); ${ }^{11}$, Fain \& Aitken (1970); ${ }^{12}$, Fain $(1972) ;{ }^{13}$, Fain $(1973 a) ;{ }^{14}$, Fain (1973b); ${ }^{15}$, Confalonieri (1976); ${ }^{16}$, Fain (1976); ${ }^{17}$, Webb JR. \& Loomis (1977); ${ }^{18}$, Whitaker JR \& Mumford (1977); ${ }^{19}$, Fain (1978); ${ }^{20}$, UChiKaWA (1987); ${ }^{21}$, Gettinger \& Gribel (1989); ${ }^{22}$, Peracchi (1990); ${ }^{23}$, Klompen (1992); ${ }^{24}$, FonseCa et al. (2005); ${ }^{25}$, Azevedo et al. (2002); ${ }^{26}$, Dantas-Torres et al. (2009); ${ }^{27}$, Silva et al. (2009); ${ }^{28}$, AlmeidA et al. (2011); ${ }^{29}$, GoMes et al. (2013); ${ }^{30}$, presente trabalho.

\begin{tabular}{lcc}
\hline Espécies de ácaros & Espécies de Hospedeiros & Estado
\end{tabular}

\section{Macronyssidae}

Chiroptonyssus haematophagus (Fonseca, 1935)

C. robustipes (Ewing, 1925)

Macronyssoides kochi (Fonseca, 1948)

Macronyssoides sp.

Macronyssus crosbyi (Ewing \& Stover, 1915)

Macronyssus meridionalis Radovksi, 1967

Macronyssus sp. 1

Macronyssus sp. 2

Mitonyssus noctilio Yunker \& Radovsky, 1980

Parichoronyssus crassipes Radovsky, 1967

P. euthysternum Radovsky, 1967

P. sclerus Radovsky, 1966

Parichoronyssus sp.

Radfordiella desmodi Radovsky, 1967

R. oudemansi Fonseca, 1948

Radfordiella $\mathrm{sp.}$

Steatonyssus joaquimi (Fonseca, 1935)

Steatonyssus sp.

\section{SPINTURNICIDAE}

Periglichrus acutisternus Machado-Allison, 1964

P. caligus Kolenati, 1857

P. herrerai Machado-Allison, 1965

P. hopkinsi Machado-Allison, 1965

P. iheringi Oudemans, 1902

P. micronycteridis Furman, 1966

P. ojastii Machado-Allison, 1964

P. paravargasi Herrin \& Tipton, 1975

P. parvus Machado-Allison, 1964

P. ramirezi Machado-Allison \& Antequera, 1971

P. tonatii Herrin \& Tipton, 1975

P. torrealbai Machado-Allison, 1965

P. vargasi Hoffman, 1944

Periglischrus sp.

Spinturnix americanus (Banks, 1902)

S. surinamensis Dusbábek \& Lukoschus, 1971

Spinturnix sp.
Cynomops abrasus $^{1}$; Eumops dabennei ${ }^{30}$; Histiotus velatus ${ }^{25}$;

Molossus molossus ${ }^{30} ;$ M. rufus ${ }^{30}$

Noctlio albiventris ${ }^{30}$; Nyctinomops macrotis ${ }^{1}$

Artibeus lituratus ${ }^{1,28} ;$ A. planirostris ${ }^{30}$; Desmodus rotundus ${ }^{7}$;

Platyrrhinus lineatus ${ }^{25,30}$; Sturnira lilium ${ }^{30}$

Artibeus lituratus ${ }^{25} ;$ A . planirostris ${ }^{30}$

Myotis ruber ${ }^{7}$

Myotis nigricans ${ }^{30}$

Chrotopterus auritus $^{30}$

Myotis nigricans ${ }^{30}$

Noctilio albiventris ${ }^{30}$

Sturnira lilium ${ }^{25}$

Sturnira lilium ${ }^{28}$

Anoura caudifer ${ }^{18}$

Artibeus lituratus $^{28}$; Phyllostomus hastatus ${ }^{30}$

Carollia perspicillata ${ }^{28}$; Desmodus rotundus ${ }^{25,30}$

Desmodus rotundus ${ }^{1}$; Phyllostomus discolor ${ }^{30}$

Artibeus lituratus ${ }^{25}$ 28; Carollia perspicillata ${ }^{25}$

Artibeus planirostris ${ }^{30}$; Glossophaga soricina ${ }^{1}$;

Myotis nigricans ${ }^{30}$

Histiotus velatus $^{18,25}$; Myotis riparius ${ }^{18}$

Mimon bennettii ${ }^{28} ;$ Phyllostomus discolor ${ }^{21,26,30} ;$ P. hastatus $^{15,30}$

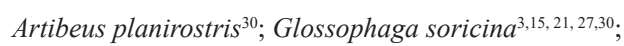

Platyrrhinus lineatus ${ }^{30}$; Não identificado ${ }^{15}$

Artibeus planirostris ${ }^{30}$; Desmodus rotundus ${ }^{15,21,28,30}$;

Myotis nigricans ${ }^{30}$; Sturnira lilium ${ }^{30}$

Rhinophylla pumilio $^{17}$

$\mathrm{RJ}^{1}, \mathrm{MG}^{25}, \mathrm{MS}^{30}$

$\mathrm{MS}^{30}, \mathrm{SP}^{1}$

$\mathrm{MG}^{25}, \mathrm{MS}^{30}, \mathrm{RJ}^{28}, \mathrm{SP}^{1,7}$

$\mathrm{MG}^{25}, \mathrm{MS}^{30}$

$\mathrm{SP}^{7}$

$\mathrm{MS}^{30}$

$\mathrm{MS}^{30}$

$\mathrm{MS}^{30}$

$\mathrm{MS}^{30}$

$\mathrm{MG}^{25}$

$\mathrm{RJ}^{28}$

$\mathrm{MG}^{18}$

$\mathrm{MS}^{30}, \mathrm{RJ}^{28}$

$\mathrm{MG}^{25}, \mathrm{MS}^{30}, \mathrm{RJ}^{28}$

$\mathrm{MS}^{30}, \mathrm{SP}^{1}$

$\mathrm{MG}^{25}, \mathrm{RJ}^{28}$

$\mathrm{MS}^{30}, \mathrm{SP}^{1}$

$\mathrm{MG}^{18,25}$

$\mathrm{DF}^{21}, \mathrm{MA}^{15}, \mathrm{MG}^{15}, \mathrm{MS}^{30}$,

$\mathrm{PE}^{26}, \mathrm{PR}^{15}, \mathrm{RJ}^{28}, \mathrm{SP}^{15}$

$\mathrm{DF}^{3,21}, \mathrm{MS}^{30}, \mathrm{MT}^{15}, \mathrm{RJ}^{15}$, $\mathrm{RS}^{27}, \mathrm{SP}^{15}$

$\mathrm{DF}^{21}, \mathrm{MS}^{30}, \mathrm{RJ}^{15,28}, \mathrm{SP}^{15}$

$?$

Anoura sp. ${ }^{27}$; Artibeus cinereus ${ }^{21}$; A. fimbriatus $^{27}$;

A. lituratus ${ }^{3,15,21,25,26,27,28,30} ;$ A. planirostris ${ }^{15,21,26,28,30} ; A$.

obscurus $^{28}$; Carollia perspicillata ${ }^{15,28}$; Chrotopterus auritus ${ }^{15}$;

Desmodus rotundus ${ }^{15}$;

Eptesicus brasiliensis ${ }^{15}$; Glossophaga soricina ${ }^{27,30}$;

Lophostoma silviculum $^{30}$; Noctilio albiventris ${ }^{30}$; Peropteryx

macrotis $^{15}$; Phyllostomus discolor ${ }^{30}$; Platyrrhinus lineatus ${ }^{3,15,21,}$

${ }^{26}$; Platyrrhinus sp. ${ }^{15}$; Sturnira lilium ${ }^{15,25,26,28,30} ;$ S. tildae ${ }^{15}$;

Vampyrodes caraccioli ${ }^{15}$

Micronycteris megalotis ${ }^{28}$

Carollia perspicillata $^{26}$; Sturnira lilium ${ }^{15,21,25,28,30} ;$ S. tildae t5 $^{15}$

Anoura caudifer ${ }^{21}$

Mycronicteris megalotis ${ }^{15}$

Rhynophylla pumilio ${ }^{15,17}$, Uroderma sp. $^{15}$

Lophostoma silvicolum $^{30}$

Lophostoma silviculum $^{30} ;$ Phyllostomus discolor $^{21,30} ;$ P.

hastatus ${ }^{15,28,30}$

Anoura caudifer ${ }^{15,27}$; A. geoffroyi ${ }^{27}$; Anoura sp. $^{27}$; Glossophaga soricina $^{15}$

$\mathrm{RJ}^{28}$

$\mathrm{DF}^{21}, \mathrm{ES}^{15}, \mathrm{MG}^{15,25}, \mathrm{MS}^{30}$, $\mathrm{PE}^{26}, \mathrm{RJ}^{15,28}$

$\mathrm{DF}^{21}$

$\mathrm{MT}^{15}$

$\mathrm{ES}^{15}$

$\mathrm{MS}^{30}$

$\mathrm{DF}^{21}, \mathrm{MG}^{15}, \mathrm{MS}^{30}, \mathrm{RJ}^{28}$, $\mathrm{SP}^{15}$

$\mathrm{RS}^{27}, \mathrm{RJ}^{15}$

Carollia perspicillata ${ }^{25}$; Nyctinomops macrotis ${ }^{15}$; Phylloderma stenopsis $^{15}$; Pygoderma bilabiatum ${ }^{15}$

Myotis albescens ${ }^{30} ;$ M. nigricans ${ }^{15,30} ;$ M. simus $^{15,30} ;$ Myotis

sp. $^{30}$; Nyctinomops macrotis ${ }^{15}$

Eptesicus braliensis ${ }^{15}$

Eptesicus brasiliensis ${ }^{15}$
$\mathrm{MA}^{15}, \mathrm{MG}^{25}, \mathrm{PA}^{15}, \mathrm{RJ}^{15}$

$\mathrm{MG}^{15}, \mathrm{MS}^{30}, \mathrm{PA}^{15}, \mathrm{RJ}^{15}$

$\mathrm{PA}^{15}$

$\mathrm{SP}, \mathrm{MG}^{15}$ 
Tab. I. Cont.

\begin{tabular}{|c|c|c|}
\hline Espécies de ácaros & Espécies de Hospedeiros & Estado \\
\hline \multicolumn{3}{|l|}{ SPELAEORHYNCHIDAE } \\
\hline Spelaeorhynchus jimi Peracchi, 1990 & Micronycteris megalotis ${ }^{22}$ & $\mathrm{MT}^{22}$ \\
\hline Spelaeorhynchus praecursor Neumann, 1902 & $\begin{array}{l}\text { Anoura caudifer }^{22} ; \text { Carollia brevicauda }^{22} ; \text { C. perspecillata } \\
\text { Glossophaga soricina }\end{array}$ & $\mathrm{MG}^{22}, \mathrm{PE}^{22,26}, \mathrm{RJ}^{22}, \mathrm{SP}^{22}$ \\
\hline Spelaeorhynchus sp. & Carollia brevicauda ${ }^{6}$ & $\mathrm{PE}^{6}$ \\
\hline \multicolumn{3}{|l|}{ TROMBIDIFORMES } \\
\hline \multicolumn{3}{|l|}{ EREYNETIDAE } \\
\hline Speleochir barbulata Fain \& Aitken, 1970 & Mimon crenulatum ${ }^{11}$ & $\mathrm{PA}^{11}$ \\
\hline S. brasiliensis Fain \& Aitken, 1969 & Artibeus planirostris $^{10}$, Vampyrodes caraccioli ${ }^{10}$ & $\mathrm{PA}^{10}$ \\
\hline \multicolumn{3}{|l|}{ LEEUWENHOEKIIDAE } \\
\hline Whartonia pachywhartoni Vercammen-Grandjean, 1966 & Micronycteris megalotis ${ }^{5}$ & ? \\
\hline $\begin{array}{l}\text { MYOBIIDAE } \\
\text { Acanthophthirius (Myotimyobia) lucifugus nigricans } \\
\text { Fain, } 1978\end{array}$ & Myotis nigricans ${ }^{19}$ & $\mathrm{RJ}^{19}$ \\
\hline Eudusbabekia diaemis Uchikawa, 1987 & Diaemus youngi $i^{20}$ & $\mathrm{MT}^{20}$ \\
\hline E. diphyllis Uchikawa, 1987 & Diphylla ecaudata ${ }^{20}$ & $\mathrm{PE}^{20}$ \\
\hline E. urodermae Fain, 1972 & Uroderma magnirostrum $^{12}$ & $\mathrm{PA}^{12}$ \\
\hline Eudusbabekia sp. & Artibeus lituratus $^{25} ;$ A. planirostris $^{30}$ & $\mathrm{MG}^{25}, \mathrm{MS}^{30}$ \\
\hline Phyllostomyobia chrotopterus Fain, 1973 & Chrotopterus sp. ${ }^{13}$ & $\mathrm{SC}^{13}$ \\
\hline \multicolumn{3}{|l|}{ TROMBICULIDAE } \\
\hline Beamerella sp. & Artibeus planirostris $^{30}$ & $\mathrm{MS}^{30}$ \\
\hline Colicus sp. & Carollia perspicillata $^{25}$, Histiotus velatus ${ }^{25}$ & $\mathrm{MG}^{25}$ \\
\hline Fonsecia sp. & Glossophaga soricina ${ }^{25}$ & $\mathrm{MG}^{25}$ \\
\hline Hooperella spinirostra Vercammen-Grandjean, 1967 & Micronycteris megalotis ${ }^{8}$ & $?$ \\
\hline Perissopalla barticonycteris Brennan, 1969 & Gliphonycteris daviesi ${ }^{9}$ & $\mathrm{PA}^{9}$ \\
\hline P. ipeani Brennan, 1969 & Carollia perspicillata $^{9}$ & $\mathrm{PA}^{9}$ \\
\hline Perissopalla sp. & Carollia perspicillata ${ }^{25}$, Desmodus rotundus ${ }^{25}$ & $\mathrm{MG}^{25}$ \\
\hline \multicolumn{3}{|l|}{ SARCOPTIFORMES } \\
\hline \multicolumn{3}{|l|}{ LABIDOCARPIDAE } \\
\hline Dentocarpus silvai silvai Dusbabek \& Cruz, 1966 & Molossus rufus ${ }^{24}$ & $\mathrm{RJ}^{24}$ \\
\hline Labidocarpellus chrotopterus Fain, 1976 & Chrotopterus auritus $^{16}$ & $\mathrm{SC}^{16}$ \\
\hline Lawrenceocarpus mimon Fain, 1970 & Mimon bennettii ${ }^{14}$ & $\mathrm{AM}^{14}$ \\
\hline Parakosa flexipes (Pinichpongse, 1963) & Molossus rufus ${ }^{29,30}$ & $\mathrm{BA}^{29}, \mathrm{MS}^{30}$ \\
\hline Parakosa rectipes (Pinichpongse 1963) & Cynomops abrasus $^{30} ;$ Molossus molossus ${ }^{30} ;$ M. rufus ${ }^{30}$ & $\mathrm{MS}^{30}$ \\
\hline Paralabidocarpus tonatiae Fain, 1970 & Sturnira lilium ${ }^{14}$ & $\mathrm{AM}^{14}$ \\
\hline Paralabidocarpus sp. & Phyllostomus hastatus ${ }^{25}$ & $\mathrm{MG}^{25}$ \\
\hline \multicolumn{3}{|l|}{ ROSENSTEINIIDAE } \\
\hline Nycterigliphus sturnirae Fain, 1963 & Sturnira lilium $^{4}$ & $\mathrm{AM}^{4}$ \\
\hline \multicolumn{3}{|l|}{ SARCOPTIDAE } \\
\hline Chirnyssoides amazonae Fain, 1959 & Carollia perspicillata $^{2}$ & $\mathrm{AM}^{2}$ \\
\hline C. brasiliensis Fain, 1959 & Sturnira lilium $^{2}$ & $\mathrm{AM}^{2}$ \\
\hline C. caparti Fain, 1959 & Artibeus planirostris ${ }^{2,30}$ & $\mathrm{AM}^{2}, \mathrm{MS}^{30}$ \\
\hline C. phyllostomus Fain \& Lukoschus, 1975 & Tonatia carrikeri $i^{23}$ & $\mathrm{PA}^{23}$ \\
\hline Chirnyssoides sp. & Carollia perspicillata $^{2}$ & $\mathrm{AM}^{2}$ \\
\hline
\end{tabular}

registradas, sendo que no Brasil as espécies Chirnyssoides caparti, Macronyssus meridionalis, Mitonyssus noctilio, Parakosa rectipes, Periglischrus tonatii e Beamerella sp. foram encontradas somente no Mato Grosso do Sul (Tab. I).

As duas famílias com maior número de espécies registradas no país são Macronyssidae e Spinturnicidae (Tab. I). No entanto, a distribuição das espécies apresenta padrões diferenciados para cada família. Os macronissídeos são conhecidos para quatro estados geograficamente próximos, enquanto espinturnicídeos foram registrados em todas as regiões do país.
Principais lacunas de conhecimento. Um dos principais problemas que impedem um aumento no conhecimento da distribuição de ácaros em morcegos no Brasil é a ausência de coleções públicas de referência e taxonomistas atuando no país. Os ácaros ectoparasitos de morcegos são frequentes, porém alguns grupos são pouco coletados, provavelmente devido seu tamanho corporal pequeno (Guerrero, 1996). Além disso, é necessária a elaboração de chaves de identificação recentes que incluam todas as espécies conhecidas, bem como a descrição dos táxons ainda não descritos. 
Perspectivas de pesquisa para os próximos 10 anos. Levantamentos taxonômicos, pesquisas sobre estrutura de comunidades e monitoramentos ambientais são atividades frequentemente desenvolvidas por pesquisadores no Brasil. Um simples procedimento que melhoraria a quantidade e qualidade dos espécimes de ácaros nas coleções seria uma coleta rotineira, com os devidos procedimentos para a obtenção desses parasitos sem riscos de falsos hospedeiros. Dentre eles, a individualização de morcegos mortos em sacos plásticos durante as atividades de campo e posterior encaminhamento para coleções científicas, nas quais técnicos e/ou pesquisadores aplicariam metodologia específica de coleta de ácaros sobre o corpo do hospedeiro.

Recomenda-se o investimento na captura desses ectoparasitos nos Chiroptera no Brasil, além da formação de taxonomistas especialistas nestes grupos de ácaros para auxiliar na correta identificação das espécies, bem como no treinamento de estudantes em todo país.

Agradecimentos. À Fundação de Apoio ao Desenvolvimento do Ensino, Ciências e Tecnologia do Estado de Mato Grosso do Sul (Fundect) e a Superintendência de Ciências e Tecnologia do Estado de Mato Grosso do Sul (Sucitec/MS) pelo convite de participação neste volume especial da Iheringia, série Zoologia e o suporte financeiro para sua publicação. Este trabalho foi parcialmente financiado pela Fundação de Amparo à Pesquisa do Estado de São Paulo (MPV: FAPESP 2011/11420-5; 2012/06951-4)

\section{REFERÊNCIAS BIBLIOGRÁFICAS}

Almeida, J. C.; Silva, S. S. P.; Serra-Freire, N. M. \& Valim, M. P. 2011. Ectoparasites (Insecta and Acari) associated with bats in Southeastern Brazil. Journal of Medical Entomology 48:753-757.

Azevedo, A. A.; Linardi, P. M. \& Coutinho, M. T. Z. 2002. Acari ectoparasites of bats from Minas Gerais, Brazil. Journal of Medical Entomology 39:553-555.

Brennan, J. M. 1969. New bat chiggers of the genus Perissopalla from Venezuela and Northeastern Brazil (Acarina: Trombiculidae). Journal of Medical Entomology 6:427-431.

Confalonieri, U. E. C. 1976. Sobre a família Spinturnicidae Oudemans, 1902 e seus hospedeiros no Brasil, com estudo biométrico de Periglischrus iheringi Oudemans, 1902 e Periglischrus ojastii Machado-Allison, 1964 (Arthropoda: Acari: Mesostigmata). Dissertação de Mestrado. Universidade Federal Rural do Rio de Janeiro. 92p.

Dantas-Torres, F.; Soares, F. A. M.; Ribeiro, C. E. B. P.; Daher, M. R. M.; ValençA, G. C. \& Valim, M. P. 2009. Mites (Mesostigmata: Spinturnicidae and Spelaeorhynchidae) associated with bats in Northeast Brazil. Journal of Medical Entomology 46:712-715.

FaIN, A. 1959. Les acariens psoriques parasites des chauves-souris. X. Le Genre Chirnyssoides g. n. chez les chauves-souris bub-americaines (Sarcoptiformes: Sarcoptidae). Bulletin de l' Institut Royal des Sciences Naturelles de Belgique 35:1-19.

FaIN, A. 1963. Les tyrogliphides commensaux des chauves-souris insectivores - Description de cinq especes nouvelles. Revue de Zoologie et de Botanique Africaines 67:33-58.

FaIN, A. 1970. Acarines nasicoles d'oiseaux et de mammiferes du Bresil. IV. Nouveaux Ereynetidae (Trombidiformes) et Turbinoptidae (Sarcoptiformes) de la region de Belem (nord Bresil). Acarologia 12:326-238.

FAIN, A. 1972. Diagnoses de nouveaux Myobiidae (Acarina: Trombidiformes). Revue de Zoologie et de Botanique Africaines 86:148-157.

FAIN, A. 1973a. Nouveaux taxa dans la famille Myobiidae (Acarina: Trombidiformes). Revue de Zoologie et de Botanique Africaines 87:614-621.

FAIN, A. 1973b. Les Listrophoridés d'Amérique Néotropicale (Acarina:
Sarcoptiformes). 1. Familles Listrophoridae et Chirodiscidae. Bulletin de l'Institut Royal des Sciences Naturelles de Belgique 49(6):1-149.

FAIN, A. 1976. Nouveaux acariens parasites de la Superfamille Listrophoroidea (Astigmates). Acta Zoologica et Pathologica Antverpiensia 64:37-67.

FAIN, A. 1978. Nouveaux Myobiidae parasites de chiropteres (Acarina: Prostigmates). Bulletin et Annales de la Societe Royale Belge d'Entomologie 114:61-76.

Fain, A. \& AitKen, T. H. G. 1969. Acarines nasicoles d'oiseaux et de mammiferes du Bresil. II. Ereynetidae de la region de Belem (nord Bresil). Bulletin et Annales de la Societe Royale Belge d'Entomologie 105:33-44.

Fain, A.; Anastos, G.; Camin, J. \& Johnson, D. 1967. Notes on the genus Spelaeorhyncus: description of $S$. praecursor Neumann and of two new species. Acarologia 9:535-556.

FonSECA, F. A. 1948. Monograph of the general and species of Macronyssidae Oudemans, 1936 (synom.: Liponyssidae Vitzthum, 1931) (Acari). Proceedings of the Zoological Society of London 118:249-334.

Fonseca, M. A.; Valim, M. P.; Вotão-Miranda, R. A.; Gitti, C. B.; Amorim, M. \& Serra-Freire, N. M. 2005. Ocorrência de Dentocarpus silvai silvai Dusbabek \& Cruz, 1966 (Acari: Chirodiscidae) em duas espécies de molossídeos (Mammalia: Chiroptera) no estado do Rio de Janeiro, Brasil. Entomología y Vectores 12(1):117-121.

Gardner, A. L. 2008. Mammals of South America: Marsupials, xenarthrans, shrews, and bats. Vol. I. Chicago, University of Chicago Press. 690p.

GetTinger, D. \& Gribel, R. 1989. Spinturnicid mites (Gamasida, Spinturnicidae) associated with bats in Central Brazil. Journal of Medical Entomology 26:491-493.

Gomes, L. A. C.; Silva, R. M. \& Melo, V. L. 2012. First record of Parakosa flexipes (Acari: Chirodiscidae) parasitizing a free-tailed bat (Chiroptera: Molossidae) in Brazil. Revista Brasileira de Parasitologia Veterinária 22:424-427.

Guerrero, R. 1996. Estudio preliminar de los ectoparasitos de los murciélagos de Pakitza, Parque Nacional Manú (Perú). In: Wilson, D. E. \& SANDOval, A. eds. Manu - The Biodiversity of Southeastern Peru. Lima, Smithsonian Institute, Editorial Horizonte, p. 643-657.

Klompen, J. S. H. 1992. Phylogenetic relationships in the mite family Sarcoptidae (Acari: Astigmata). Miscellaneous Publications Museum of Zoology, University of Michigan 180:1-155.

Krantz, G. W. \& Walter, D. E. 2009. A Manual of Acarology. 3ed. Lubbock, Texas Tech University Press. 807p.

Marshall, A. G. 1981. Ecology of ectoparasitic insects. London, Academic Press. 459p.

PeracChI, A. L. 1990. Contribuição ao estudo da família Spelaeorhynchidae Oudemans, 1902 (Acarina, Mesostigmata). Revista Brasileira de Zoologia 7(1-2):1-29.

RADOVSKY, F. J. 1967. The Macronyssidae and Laelapidae (Acarina, Mesostigmata) parasitic on bats. University of California Publications in Entomology 46:1-288.

RUDNICK, A. 1960. A revision of the mites of the family Spinturnicidae (Acarina). University of California Publications in Entomology 17:157-284.

Silva, C. L.; Graciolli, G. \& RuI, A. M. 2009. Novos registros de ácaros ectoparasitos (Acari, Spinturnicidae) de morcegos (Chiroptera, Phyllostomidae) no Rio Grande do Sul, Brasil. Chiroptera Neotropical 15:469-471.

UCHIKAWA, K. 1987. Myobiid mites (Trombidiformes: Myobiidae) associated with the vampire bats (Chiroptera: Phyllostomatidae) and information on host taxonomy deduced from them. Journal of Parasitology 73:640645.

Vercammen-Grandjean, P. H. 1966. Whartonia pachywhartoni $\mathrm{n}$. sp., an extraordinary parasite of a Brazilian bat (Leeuwenhoekiidae-Acarina). Acaralogia 8:282-284

VerCammen-Grandjean, P. H. 1967. Revision of the genus Tecomatlana Hoffman, 1947 (Acarina: Trombiculidae). Acaralogia 9:848-864.

Webi JR., J. P. \& Loomis, R. B. 1977. Ectoparasites. In: BaKer, R. J.; Knox Jones JR., J. \& CARTER, D. C. ed. Biology of Bats of the New World Family Phyllostomatidae. Lubbock, The Museum Texas Tech University. (Special Publications 13. Part II), p. 57-119.

WhitAKER JR., J. O. \& Mumford, R. E. 1977. Records of ectoparasites from Brazilian mammals. Entomological News 88(9-10):255-258. 